\title{
Indirekte Evidentialität in der lateinischen erzählenden Prosa des I-II Jhdt.
}

Bohdan Chernyukh

(Ivan Franko National University of Lviv)

Indirect Evidentiality in Latin Narrative Prose of I-II A. D.

\begin{abstract}
The paper examines the means of expressing indirect (inferentive and reportative) evidentiality in Latin narrative prose of I-II A. D. on the material Petron's Satyricon and Apuleius' Metamorphoses. Morphological forms and syntactic constructions treated as evidential strategies are taken into account, since their main meanings are within the pattern of the grammatical categories (tense, mood etc.), and the information about the source and/or means of access to information is contextually motivated side effect. The result of the research reveals singling out eight strategies to express indirect evidentiality. Inferentive evidentiality is represented by five evidential strategies, while the reportative - by the three. Different use of evidential strategies in both works relates to context and personal choice of the authors.
\end{abstract}

\section{Keywords}

indirect evidentiality; evidential strategies; inferentive and reportative evidentiality; Petron; Apuleius 


\section{Einführung}

Seit den 80er Jahren des vorigen Jahrhunderts gehört die Kategorie der Evidentialität, die sich auf die Informationsquelle bzw. auf die Weise des Zugangs zur Information bezieht, ${ }^{1}$ auf die als erster R. Jakobson aufmerksam gemacht hat, ${ }^{2}$ zu den aktuellen Themen der heutigen Sprachwissenschaft.

Der Weise nach, wie man eine Information kriegt, werden drei Haupttypen der Evidentialität unterschieden: direkte Evidentialität (visuelle, auditive, andere sensorielle), indirekte inferentive Evidentialität (auf Grund einer logischen Schlussfolgerung bzw. einer Vermutung) und indirekte reportative Evidentialität (der zugrunde die Worte einer anderen Person liegen). ${ }^{3}$ Die genannten Haupttypen werden ihrerseits in Untertypen

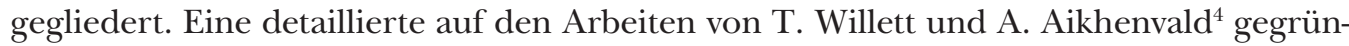
dete Klassifikation der evidentiellen Bedeutungen ist von V. Plungian ${ }^{5}$ vorgeschlagen worden.

In allen erwähnten Klassifikationen ist Unterscheidung zwischen der direkten und indirekten Evidentialität grundliegend. Bei der direkten Evidentialität handelt es sich um einen unmittelbaren Zugang eines Sprechers bzw. eines Hörers zur Informationsquelle anhand der Sinnesorgane oder durch die unmittelbare Teilnahme an einer Situation.

Die indirekte Evidentialität (inferentive und reportative) je nach der Stufe der Heranziehung eines Sprechers wird auf die persönliche und unpersönliche gegliedert. Die inferentive Evidentialität ist persönlich: der Sprecher macht eine Schlussfolgerung über die Situation, an der er sich nicht beteiligt hat, aufgrund des eigenen Urteils. Die reportative Evidentialität dagegen ist unpersönlich, da die Informationsquelle außer dem Sprecher liegt. ${ }^{6}$

Sowohl die Anzahl der evidentiellen Bedeutungen als auch ihre Verbindlichkeit bzw. Fakultativität und Ausdrucksmittel variieren sich der Sprache nach. Einige Sprachen verfügen über besondere Marker der Evidentialität (Affixe, Klitika usw.), während in den anderen ihre Ausdrucksmittel mit Markern der grammatischen Kategorien (z.B. Tempus, Modus, Aspekt) verschmolzen sind. ${ }^{7}$ Es ist aber zweifellos, dass solche Mittel in jeder Sprache vorhanden sind, und zwar unterschiedliche. Außer der morphologischen

1 Aikhenvald (2004: p. 3; 2011: p. 605).

2 Die theoretischen Grundlagen der Evidentialität sind von R. Jakobson in seinem Aufsatz Shifters, Verbal Categories, and the Russian Verb (1957) formuliert worden: "Evidential is a tentative label for the verbal category which takes into account three events - a narrated event, a speech event, and a narrated speech event, namely the alleged source of information about the narrated event," Jakobson (1984: p. 46).

3 Jakobson (1984: p. 46): “The speaker reports an event on the basis of someone else's report (quotative, i.e. hearsay evidence), of a dream (revelative evidence), of a guess (presumptive evidence) or on his own previous experience (memory evidence)."

4 Willett (1988); Aikhenvald (2004).

5 Plungian (2010: p. 37); vgl. Aikhenvald (2018: p. 47).

6 Plungian (2010: pp. 28-34, passim); van Rooy (2016: p. 8).

7 Die speziellen Marker der Evidentialität sind in den Sprachen des Nord- und Südamerikas, tibeto-birmanischen, balkanischen und einigen anderen Sprachen vorhanden, siehe Aikhenvald (2004: pp. 23-66); Aikhenvald \& Dixon (1998: p. 245). 
Marker, die zuerst als ausschließliche Merkmale der Evidentialität betrachtet wurden, wird in der letzten Zeit mehr Aufmerksamkeit auch anderen, weniger grammatisierten und sogar lexikalischen Mitteln geschenkt. ${ }^{8}$ Als solche können z.B. 'anscheinend' und 'wahrscheinlich' im Deutschen, 'perhaps' und 'seemingly' im Englischen, 'ecce' und 'fortasse' im Latein gemeint werden. Die Natur solcher Mittel, ob sie zur Grammatik oder zur Lexik gehören, wird in vielen modernen Arbeiten über die Evidentialität behandelt. ${ }^{9}$

Wenn es sich um die Ausdrucksmittel der Evidentialität geht, werden drei Gruppen der Sprachen unterschieden, und zwar:

1) Sprachen mit der grammatisierten Evidentialität;

2) Sprachen mit der lexikalisierten Evidentialität;

3) Sprachen mit kumulativen Markern der Evidentialität. ${ }^{10}$

Um die letzten Ausdrucksmittel zu bezeichnen hat A. Aikhenvald einen passenden Fachbegriff 'evidentielle Strategien' (evidential strategies) vorgeschlagen. ${ }^{11}$ Darunter werden die Formen und Konstruktionen ohne direkten Bezug auf Informationsquelle gemeint, ${ }^{12}$ deren Grundbedeutungen auf der Ebene der anderen grammatischen Kategorien (Tempus, Modus usw.) liegen. $\mathrm{Zu}$ solchen evidentiellen Strategien in einigen Sprachen gehören Futurformen, modale und syntaktische Konstruktionen usw., bei denen die Evidentialität als ein Nebeneffekt (side effect) in einem bestimmten Kontext entsteht. ${ }^{13}$

Die Existenz solcher Strategien wird dadurch verursacht, dass nicht alle Sprachen über die spezifischen Ausdrucksmittel der Evidentialität verfügen, während jede Sprache sowieso eine Informationsquelle ausdrückt. Die Grenze zwischen grammatischen und lexikalischen Ausdrucksmitteln der Evidentialität ist nicht scharf und lässt das Vorhandensein einer gewissen Zwischenzone zu. Wie B. Wiemer betont, sei der Unterschied zwischen grammatischen und lexikalischen Mitteln nicht bipolar, aber er solle eher als ein allmähliches Kontinuum betrachtet zu werden, das sich von 'maximal grammatisierten' (highly grammaticalized) Mitteln zu den 'lexikalischen' (lexical) über 'weniger grammatisierte' (less grammaticalized) ausstreckt. ${ }^{14}$

8 Aikhenvald \& Dixon (2003); Diewald \& Smirnova (2010); Cornillie (2007a); Hennemann (2013).

9 Über verschiedene Meinungen zu diesem Problem siehe Boye \& Harde (2009: pp. 9-14).

10 Lazard (2001: p. 360); Aikhenvald (2018: pp. 39-40). Vgl. Squartini (2008: p. 918): "Lazard ... as well as Aikhenvald ..., also emphasizes the importance of maintaining a sharp distinction between grammatical and lexical expressions of evidentiality, the latter possibly being a universal feature represented by lexical elements such as the English adverbs reportedly, allegedly, evidently, etc."

11 Aikhenvald (2004: pp. 105-152).

12 Aikhenvald (2003: p. 2).

13 Aikhenvald (2011: p. 606): "For each of these forms, information source is just one of their meaning extensions, a 'side-effect'. Categories and forms which have additional meanings to do with information source are known as 'evidentiality strategies'." Vgl. Friedman (1986: p. 185): "(evidential strategies) do not mark the source of information or evidence, but rather the speaker's attitude toward it. The question of whether the source of information was a report, deduction, direct experience or something else is answered by the context in which the speaker's choice of form occurs."

Wiemer (2010: p. 63). 
Das Vorhandensein eines solchen Kontinuums, das verschiedenartige Mittel umfasst, neigt immer mehr Forscher die Evidentialität als eine funktionell-semantische Sphäre zu betrachten, die nicht ausschließlich durch grammatikalische Merkmale gekennzeichnet ist. ${ }^{15}$ G. Lampert \& M. Lampert schlagen vor, die Evidentialität als eine multidimensionale kontextuelle Kategorie anzusehen, die alle auf sie im Kontext hinweisenden sprachlichen Repräsentationen umfasst. ${ }^{16}$

Die Evidentialität und ihre Ausdrucksmittel in den Weltsprachen werden in mehreren Untersuchungen behandelt. ${ }^{17}$

Trotz einem gewissen Skeptizismus über die Existenz der Evidentialität in den "toten Sprachen"18 sind in den letzten zwei Jahrzehnten einige evidentiell-orientierte Untersuchungen auf dem Gebiete der klassischen Sprachen erschienen. Einige Aspekte der Evidentialität im Lateinischen haben P. Cuzzolin, P. Greco, C. Guardamagna, E. Zheltova erforscht. ${ }^{19}$ Die Existenz der Evidentialität im Indogermanischen zu begründen hat B. Joseph versucht. ${ }^{20}$ Es gibt auch Arbeiten, die die Evidentialität im Altgriechischen behandeln. ${ }^{21}$

Wenn es sich um das Lateinische handelt, kann man hier wie in den anderen Sprachen das Vorhandensein der direkten und indirekten Evidentialität behaupten, die mit lexikalischen und grammatischen (morphologischen und syntaktischen) Mitteln ausgedrückt wird. ${ }^{22}$ Wegen des Fehlens der Marker im Lateinischen, deren einzelne Funktion im Hinweis auf eine Informationsquelle besteht, soll es hier eher von den Evidentialitätsstrategien die Rede sein.

In dem vorgeschlagenen Aufsatz werden die Evidentialitätsstrategien für den Ausdruck der indirekten Evidentialität in lateinischer erzählender Prosa des I.-II. Jhdt. und zwar in Werken von Petron (Satyricon) und Apuleius (Metamorphosen) behandelt. Dabei wird die Aufmerksamkeit nur den grammatischen und grammatisierten Mitteln geschenkt. Die lexikalischen Mittel als auch die direkte Evidentialität können den Gegenstand einer weiteren Studie bilden.

15 Diewald \& Smirnova (2010: p. 1).

16 Lampert \& Lampert (2010: p. 319).

17 Z. B. Chafe \& Nichols (1986); Aikhenvald \& Dixon (2003); Diewald \& Smirnova (2010); Aikhenvald (2010); Khrakovskiy (2007).

18 Mišeska Tomić (2008: p. 212).

19 Cuzzolin (2010); Greco (2013); Guardamagna (2017); Zheltova (2017); Zheltova (2018).

20 Joseph (2003).

21 Méndez Dosuna (1999); Faure (2010); Faure (2014); Bakker (1997); Bakker (2002); van Rooy (2016); Kratschmer \& Heijnen (2010).

22 Cuzzolin (2010: pp. 250-251): “... in Sprachen, in denen die Evidentialität nicht systematisch ausgedrückt wird, ist genau die Interferenz der Evidentialität mit der Morphologie und eventuell mit der Syntax, was sich als interessant erweist und weiterer Untersuchungen wert ist. Das ist genau der Fall des Lateinischen ... Das Lateinische gehört zweifellos jenen Sprachen an, die evidentiale Merkmale meistens im Lexikon ausdrücken. Es gibt dennoch Morpheme, die besonders häufig eine pragmatische Erweiterung in Richtung nach der Evidentialität zu erlauben scheinen." 


\section{Inferentive Evidentialität}

Wie erwähnt, bezeichnet die indirekte Evidentialität eine Schlussfolgerung oder eine Vermutung des Sprechers, die sich auf den Spuren einer vorigen Situation beruhen. ${ }^{23}$ Durch analytisches Lesen der genannten Werke von Petron und Apuleius sind die folgenden Evidentialitätsstrategien festgestellt.

\subsection{Nominativus cum infinitivo}

Eine der gewöhnlichen Evidentialitätsstrategien, die die indirekte Evidentialität markiert, ist eine von dem Verb videri 'scheinen' abhängige Konstruktion Nominativus cum infinitivo (NcI), die eine Vermutung oder eine Annahme des Sprechers ausdrückt (1a-c). ${ }^{24}$

1a) ... producta est puella satis bella et quae non plus quam septem annos habere videbatur... (Petron. Sat. 25,2); “...es ist ein ziemlich schönes Mädchen vorgeführt worden, das nicht mehr als sieben Jahre alt zu sein schien...”

1b) Habinnas sevir est idemque lapidarius, qui videtur monumenta optime facere (Petron. Sat. 65,5); "Habinnas ist einer der Sechsmänner und derselbe Steinmetz, der die Denkmäler am besten zu verfertigen scheint."

1c) ... illi, qui talia vobis adfirmant, non videntur mihi mendacium fingere (Apul. Met. 5,18); “...diejenige, die euch solches sagen, scheinen mir keine Lüge zu erdichten.”

In den angeführten Beispielen bezeichnet videri die Schlussfolgerung, die der Sprecher aufgrund ihm bekannten Tatsachen macht. Dabei ist dieses Verb kein Hauptprädikat der Proposition und eben sein solcher Status ist eine der Voraussetzungen für die Identifizierung der archetypischen evidentiellen Bedeutungen. ${ }^{25}$ Es kann hier auch über die Verflechtung der Evidentialität und der epistemischen Modalität Rede sein, da die Grenzen zwischen ihnen oft unscharf $\operatorname{sind}^{26}$

Die ähnliche Konstruktion mit dem Verb paivetal zum Ausdruck der inferentiven Evidentialität findet man auch im Altgriechischen ${ }^{27}(2 \mathrm{a})$.

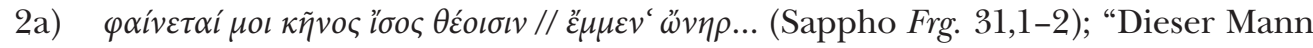
scheint mir ähnlich den Göttern zu sein...”

Es lohnt sich zu merken, dass die Verben mit ähnlichen Bedeutungen wie bei dem lateinischen Verb videri oder altgriechischen Verb paivoua können auch die gleichen Konstruktionen in den modernen Sprachen (sog. seem-constructions) bilden (2b-c).

2b) engl. "He seems to know the answer."

23 Vgl. Aikhenvald (2011: p. 605): “an inference based on general knowledge or visual traces."

24 Die statistischen Data über die Anwendungshäufigkeit der entsprechenden Ausdrucksmittel sind in Tabelle 1 angeführt.

25 Anderson (1986: pp. 274-275): "Evidentials are not themselves the main predication of the clause, but are rather a specification added to a factual claim about something else.” Vgl. Aikhenvald (2003: p. 24).

26 Plungian (2010: p. 46). Sieh Fussnote 53.

27 Vgl. van Rooy (2016: p. 38). 
2c) dt. "Er scheint die Antwort zu kennen."

Für solche Verben bzw. Konstruktionen ist in der Regel eine subjektive Interpretation eigen, ${ }^{28}$ was sie als Merkmale der inferentiven Evidentialität anzusehen erlaubt, jedoch spielt der Kontext bei ihrer Bildung eine entscheidende Rolle. ${ }^{29}$

Die inferentive Bedeutung von videri in dem angeführten Beispiel, wie sein Gebrauch als verbum regens bei NcI, wird durch Kontext verursacht, der zeigt, aus welchem Grund eine Vermutung, eine Annahme oder eine Schlussfolgerung gemacht wird.

\subsection{Coniunctivus potentialis}

Zu den verbreiteten Ausdrucksmitteln der inferentiven Evidentialität bei den erwähnten Autoren ist auch der coniunctivus potentialis zu nennen, der unter anderem eine Vermutung bzw. eine Annahme ausdrücken kann ${ }^{30}$ (3a-c). In den analysierten Werken ist er fast gleich zwischen beiden Schriftstellern verteilt.

3a) ... occurrit mihi aeque lassus ac moriens Ascyltos: putares ab eadem anicula esse deductum (Petron. Sat. 7,4); “... mir ist Ascyltus halbtot vor Müde entgegengelaufen; du würdest glauben er sei von demselben alten Mütterchen herabgeführt.”

3b) Exhorruit Tryphaena et: Putes, inquit, una nos dormisse; nam et mihi simulacrum Neptuni ... videbatur dicere: 'In nave Lichae Gitona invenies' (Petron. Sat. 104,2); "Tryphäne ist erschaudert und hat gesagt: 'Du würdest glauben, dass wir zusammengeschlafen haben. Da Neptunstatue schien auch mir zu sagen: Du wirst Giton auf dem Schiff von Lichas finden'."

3c) puer ... insurgit inque flexibus tortuosis enervam et exossam saltationem explicat .... diceres dei medici baculo, quod ramis semiamputatis nodosum gerit, serpentem generosum lubricis amplexibus inhaerere (Apul. Met. 1,4); "Ein Junge ... erhebt sich und biegt sich, als ob er keine Knochen und Muskeln hätte, zeigt einen Tanz; du würdest sagen, dass die edle Schlange um den knotigen Stock mit den halb beschnittenen Ästen, den der Gott der Ärzte trägt, mit den schlüpfrigen Umschlingungen herumschlingt."

In allen angeführten Beispielen gründet sich die durch Konjunktiv ausgedrückte Deduktion auf dem Sachverhalt, der im vorhergehenden Kontext vorhanden ist ${ }^{31}$. In der Regel wird in dieser Funktion coniunctivus imperfecti, seltener coniunctivus praesentis oder perfecti gebraucht. Es lohnt sich zu betonen, dass die inferentive Schattierung des

28 Cornillie (2007b: p. 124); Cuzzolin (2010: p. 255); vgl. de Haan (2007: p. 146).

29 Lampert \& Lampert (2010: p. 316): “[seem ] ... may become an evidential marker if one draws upon the relevant context, functioning as an attentional cueing device toward the contextually sanctioned meaning of the construction in which seem is a component."

30 Als Bedeutungen des potentiellen Konjunktivs nennen Ernout \& Thomas (1964: p. 237) Annahme (supposition), Unbestimmtheit (indefini) und gemilderte Behauptung (affirmation attenueé).

31 ÄhnlichesfindetmanauchbeianderenAutoren,z.B.iniussusignareferunt, maestique-crederesuictos-exsecrantes nunc imperatorem, nunc nauatam ab equite operam, redeunt in castra (Liv. 2. 43. 9). Vgl. Zheltova (2017: p. 325). 
potentiellen Konjunktivs fast überall auf die Formen der 2. Person beschränkt wird, was eine Distanzierung von der beschriebenen Situation unterstreicht. ${ }^{32}$ Nur einmal kommt der Konjunktiv in Form der 1. Person (3d) vor, was durch den Einfluss der Formel pace tua dixerim zu erklären ist, die 'den isolierten idiomatischen Ausdruck' (isolated idiomatic expression) $)^{33}$ darstellt.

3d) hic apud nos ... mercedes opimas iam consecutus fortunae scaevam an saevam verius dixerim miser incidit (Apul. Met. 2,13); "Auch bei uns ist er ..., obwohl er einen guten Lohn gekriegt hatte, in Armseligkeit, ich würde sagen genauer, in Nichtigkeit geraten."

Neben dem absoluten Gebrauch kommt der Konjunktiv mit evidentiellen Obertönen auch in den Konsekutivsätzen vor (4a-b).

4a) Haec ipsa cum diceret, tanta gratia conciliabat vocem loquentis, tam dulcis sonus ... mulcebat aera, ut putares inter auras canere Sirenum concordiam (Petron. Sat. 127,5); "Als sie selbst das redete, füllte so eine Anmut die Stimme der Redenden an, so ein angenehmer Schall ... streichelte die Luft, dass du glauben würdest, dass die Lüftchen einen abgestimmten Syrenenchor herbeibringen."

4b) Lignorum vero tanto me premebat pondere (puer), ut fascium molem elephanto, non asino paratam putares (Apul. Met. 7,17); “(Der Junge) überlud mich dermaßen mit Holz, so dass du glauben würdest, dass diese Bündel für einen Elefant und nicht für einen Esel bestimmt seien.”

Ein solcher Konjunktiv, sei er absolut oder im Nebensatz gebraucht, drückt eine gewisse Unbestimmtheit des Gesagten aus, was für die Information aus der zweiten Hand ${ }^{34}$ typisch ist. Die Fragestellung über die Wahrscheinlichkeit der Situation impliziert das Nichtvorhandensein einer direkten Informationsquelle und gehört somit zur indirekten Evidentialität. ${ }^{35}$

Da Coniunctivus potentialis vor allem die modalen und keine evidentiellen Bedeutungen ausdrückt, die nur kontextuell bedingt sind, ist er kein spezieller Marker der Inferentialität. Als Ausdruck einer subjektiven Meinung des Sprechers gehört ein solcher Konjunktiv zur epistemischen Modalität, ${ }^{36}$ mit welcher die Evidentialität sich kreuzt. ${ }^{37}$ Das Verhältnis zwischen der Evidentialität und der epistemischen Modalität charakterisiert V. Plungian folgenderweise: "If we regard such values as modal, we stress one of the

32 Über den Zusammenhang zwischen Person und Evidentialität siehe Kozinceva (2007: p. 17).

33 Pinkster (1986: p. 152). Das ist der einzige Fall des Gebrauchs des coniunctivus perfecti für den Ausdruck der inferentiven Evidentialität in den analysierten Werken.

34 Aikhenvald (2011: p. 610): "Non-indicative modalities may develop overtones of uncertain and nonfirsthand information."

35 Plungian (2001: p. 354).

36 Vgl. Fonseca (2009: p. 445): "Modalidad como expresión del grado de certeza del hablante sobre su enunciado, en torno, básicamente, a dos dimensiones: una 'epistémica', en la que caben juicios sobre lo cierto o incierto (posible, probable, dudoso, etc.), y otra 'deóntica', focalizada en la obligación, el mandato, el consejo, etc."

37 Über das Verhältnis der epistemischen Modalität und der Evidentialität gibt es eine umfangreiche Literatur, z.B. Givon (1982); Chafe (1986: p. 262); Willett (1988: p. 52); de Haan (1999); Mushin (2001); Cornillie (2009); Plungian (2010: pp. 44-46); Wiemer (2010: p. 60); Wiemer (2017). 
basic characteristics of modality, namely the assessment of a situation (as highly probable); regarding it as evidential, we stress one of the basic characteristics of evidentiality, namely the reference to logical conclusions as a source of information about a situation. This way, markers of presumptive evidentiality are the only evidential markers with inbuilt modal components and the only modal markers with inbuilt evidential components." 38

Der Gebrauch der morphologischen Mittel mit primärer Modalsemantik für den Ausdruck der inferentiven Evidentialität ist keine Besonderheit des Lateinischen. Ähnliche Formen sind auch in den modernen europäischen Sprachen vertreten, z.B. Konditional im Französischen ${ }^{39}$ und Italienischen, ${ }^{40}$ oder Konjunktiv im Deutschen. ${ }^{41}$

\subsection{Perfectum logicum}

In mehreren Untersuchungen wird das Perfekt im Zusammenhang mit Evidentialität erwähnt. ${ }^{42}$ Diese seine Eigenschaft wird durch die ihm eigene resultative Semantik verursacht. Die Primärfunktion des Perfekts besteht in der Bezeichnung eines Zustandes, der oft als Ergebnis einer vorigen vollendeten Handlung aufgefasst wird und im Grunde der Inferenz liegen die Spuren dieser Handlung. B. Comrie erklärt den Zusammenhang des Perfekts und der inferentiven Evidentialität wie folgend: "With the perfect, a past event is ... not simply presented per se but because of its relation to a present state. With the inferential, the past event is again not presented simply per se, rather it is inferred from some less direct result of the action. Thus the semantic similarity between perfect and inferential lies in the fact that both categories present an event not in itself, but via its results..." 43

Es ist natürlich übertrieben jedem lateinischen Perfekt die evidentiellen Obertöne zuzuschreiben, da es bei weitem nicht immer mit Gegenwart zusammenhängt. Wie bekannt ist das lateinische Perfekt das synkretische Tempus, das die formellen Merkmale und Bedeutung des Aoristus und des Perfekts im engeren Sinne verbindet. Dadurch kann es sowohl präteritale (perfectum historicum) als auch resultative (perfectum praesens oder logicum) Bedeutungen haben. Das letzte eben kann im bestimmten Kontext als Träger der Inferentivität betrachtet werden.

Die durch Perfekt ausgedrückte inferentive Evidentialität ist nur bei Apuleius (5a-b) nachgewiesen.

38 Plungian (2010: p. 46).

39 Aikhenvald (2011: p. 610); Dendale (1993); Guentchéva (1994).

40 Squartini (2001).

41 Aikhenvald (2004: p. 107); Hansen (2007: pp. 243-246).

42 Z.B. Aikhenvald (2004: p. 112); Kozinceva (2007: p. 22); Wiemer (2010: p. 66).

43 Comrie (1976: p. 110). Vgl. Aikhenvald (2004: p. 112): "The primary meaning of perfect is to focus on results of an action or process, thus relating a past event to present time. In other words, an event or a process is viewed as completed in the past but still relevant for the present. An inference is made based on some traces or results of a previous action or state. Hence there is asemantic link between a non-firsthand evidential and a perfect." 
5a) Pereo ..., immo iam dudum perii, nisi tu propitiaris (Apul. Met. 2,10); "Ich gehe verloren ... ich bin schon verloren, wenn du dich nicht erbarmst."

5b) Occisa sum misera: ... me trepidatio simul et festinatio fefellit et pyxidum similitudo decepit (Apul. Met. 3,25); "Ich, unglückliche, bin zugrunde gegangen! In Eile und Angst habe ich mich vergriffen und die Ähnlichkeit der Büchsen getauscht.”

In den angeführten Beispielen liegt die Bewertung der Ergebnisse einer vorigen Situation den von den Sprechern gemachten Schlüssen zugrunde. ${ }^{44}$ Besonders klar ist es in (5b), wo der Autor perfectum passivi gebraucht und die vorgehenden Ereignisse durch perfectum historicum (fefellit, decepit) ausgedrückt hat. ${ }^{45}$ Im Vergleich zu der aktiven Form des Perfekts bekommt das Passivperfekt öfters eine resultative Bedeutung insbesondere bei absolut gebrauchten mono- und bivalenten Verben. ${ }^{46}$ Wie A. Aikhenvald bemerkt, "a prototypical passive involves focusing attention on the original object ... and also focusing on the state it is in, as a result of the action... As a consequence, passives often have resultative connotations... Not unexpectedly, passives acquire the same evidential extensions as do resultatives." ${ }^{47}$

Es ist gleichzeitig zu betonen, dass die resultative Bedeutung des Perfekts in großer Masse vom Kontext abhängt und nicht auf Grund einer "logischen Deduktion" festgestellt werden soll. ${ }^{48}$

\subsection{Futurum primum}

Die inferentive Evidentialität wird auch manchmal durch das modal gebrauchte Futur I ausgedrückt, wenn es sich um die Unsicherheit oder die Vermutung des Sprechers handelt. Dabei ist er dem potentiellen Konjunktiv nah. ${ }^{49}$ In Satyrikon und Metamorphosen ist solches Futur fünfmal bezeugt, z.B. (6a-b):

6a) ... manum ... demisit in sinum et pertractato vasculo tam rudi: "Haec," inquit, "belle cras in promulside libidinis nostrae militabit” (Petron. Sat. 24,7); “...sie hat die Hand ... unter sein Gewand sinken lassen und nachdem sie ein so junges Gefäßchen betastet hatte, sagte sie: 'Das wird morgen als Vorspeise für unsere Lust dienen'."

6b) “Quam largus” inquit "imber aderit crastino" et percontanti marito qui comperisset istud respondit sibi lucernam praedicere (Apul. Met. 2,12); "Was für starken Regen

44 Übrigens handelt es sich in beiden Fällen um eine Quasi-Resultativitat, da hier keine echten, sondern die eingebildeten Resultate vorhanden sind, die nur in Vorstellung der handelnden Personen existieren.

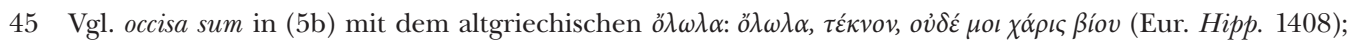
"Kind, ich bin verlorengegangen und es gibt mir keine Freude im Leben."

46 Pinkster (2015: p. 447).

47 Aikhenvald (2004: p. 116).

48 Serbat (1980); vgl. Delbrück (1897: p. 316).

49 Kühner \& Stegmann (1992: p. 142): "Die futurische Bedeutung eines Verbs geht leicht in die der Vermutung über; daher erklärt es sich, dass das Futur, namentlich in der älteren Sprache, gelegentlich in potentialem Sinne gebraucht wird." Vgl. Pinkster (2015: p. 426) mit Hinweis auf die weitere Literatur. 
werden wir morgen kriegen, sagte sie. Und nachdem der Mann sie gefragt hatte, woher sie das wisse, antwortete sie, die Lampe habe es ihr vorhergesagt."

In beiden zitierten Passagen folgen die Schlüsse der Sprecher über die behandelte Situation aus dem im naheliegenden Kontext erwähnten Nachweis: 'pertractato vasculo' (6a) und 'lucernam praedicere' (6b).

Auf die Annäherung des potentiellen Konjunktivs und des Futurs I weist ihre parallele Anwendung im Rahmen eines und desselben Satzes (6c) hin, wo sie in gleicher Bedeutung gebraucht werden.

6c) Sub extrema saxi margine poma et uvae faberrime politae dependent ... Putes (coni. praes.) ad cibum inde quaedam ... posse decerpi, et si fontem, qui deae vestigio discurrens in lenem vibratur undam, pronus aspexeris, credes (fut. I) illos ... nec agitationis officio carere (Apul. Met. 2,4); "Am Rand des Felsens hingen künstlerisch gearbeitete Äpfel und Weintrauben herab ... du würdest glauben, dass einige davon abgerissen werden können, um sie zu schmecken und wenn du dich über die Quelle hinneigst, welche unter dem Fußtritte der Göttin entspringt und rieselnd sich weiter ergießt, wirst du glauben, dass sich diese Weintrauben wie die echten bewegen."

Als Evidentialitätsstrategien treten die Futurformen auch in anderen Sprachen auf. ${ }^{50}$

\subsection{Debeo + Infinitiv}

Die Konstruktion debeo + Infinitiv zum Ausdruck der inferentiven Evidentialität wird nur bei Petron nachgewiesen $(7 \mathrm{a}-\mathrm{b})$.

7a) Plane ... hic debet servus esse nequissimus (Petron. Sat. 49,7); "Der soll ... genau der nichtsnutzigste Sklave sein.”

7b) Videtis ... mulieris compedes ... Sex pondo et selibram debet habere (Petron. Sat. 67,7); "Da seht ihr die Fesseln (d. h. Schmuck) der Frau ... Sechs und ein halbes Pfund soll sie haben."

H. Pinkster bemerkt, es handle sich dabei um 'gemilderte' Bedeutung des Verbs debeo und seine Anwendung in einer deduktiven Funktion, ${ }^{51}$ was erlaubt, darin den Marker der inferentiven Evidentialität zu ersehen.

Die ähnlichen Konstruktionen findet man auch in den anderen Sprachen, z.B. ' $m u s t$ ' im Englischen, 'müssen' im Deutschen, 'devoir' im Französischen, vgl. (7b) und seine Übersetzungen $(8 \mathrm{a}-\mathrm{c})$ :

8a) "There ... are a woman's fetters ... She must have six pounds and a half of gold on her." (übers. M. Heseltine).

8b) "Ihr sehet hier... ihre guten Fußketten! ... Sie müssen sechs und ein halbes Pfund haben." (übers. W. Heinse).

50 Aikhenvald (2011: p. 610) mit Hinweis auf die weitere Literatur; Wiemer (2010: p. 75).

51 Pinkster (2015: p. 440). 
8c) "Voyez ... l'attirail d'une femme ... Six livres de poids et la demie, c'est ce que le bracelet doit avoir." (übers. M. Nisard).

Der Grund solche Modalverben als Evidentialitätsstrategien zu betrachten, liegt darin, dass sie in den grammatischen Konstruktionen eine zusätzliche Konnotation bekommen, die eine Informationsquelle anbetrifft. ${ }^{52}$ Das schließt aber nicht mehrere Fälle aus, wo debeo (mit oder ohne Infinitiv) zum Ausdruck der Modalität dient, ${ }^{53}$ die ähnlich der Evidentialität vom Kontext abhängig ist. Deontische Modalität, z.B. ist in folgenden Beispielen (8d-e) vertreten.

8d) ... neque enim tam magnum facinus admisimus, ut debeamus torti perire (Petron. Sat. 20,1); “... wir haben kein so großes Verbrechen begangen, so dass wir durch die Qual umkommen sollen.”

8e) Non modo ... exspoliationum praedarumque, verum etiam voluptatum vestrarum ducem me strenuum sentire debetis (Apul. Met. 7,11); "Sie sollen mich nicht nur für einen rüstigen Anführer bei euren Auszügen und Räubereien, sondern auch bei eurem Vergnügen halten."

Die evidentiellen Systeme, die evidentielle und epistemische Bedeutungen verbinden, werden von V. Plungian ${ }^{54}$ als "modalisierte" (modalized evidential systems) charakterisiert. Die Nähe der Evidentialität und der epistemischen Modalität erklärt der Gelehrte dadurch, dass in beiden Fällen "the speakers do not intend to become personally convinced of the fact a situation takes or took place, but consider it as highly credible, due to certain cause-and-effect relations known to them [...] The existence of a marker of epistemic necessity is therefore, if taken for itself, not an indicator for the presence of the grammatical expression of evidentiality within the system of a language. However, markers of this kind always exhibit an intersection of modal and evidential values." 55

\section{Reportative Evidentialität}

Die reportative Evidentialität ist in beiden Werken durch die indirekte Rede vertreten, die als universelle Strategie gilt ${ }^{56}$ und mit Hilfe der folgenden Strategien ausgedrückt wird.

52 Aikhenvald (2004: p. 150).

53 Cuzzolin (2010: p. 254): "Normalerweise schwankt die Interpretation des Verbs debere zwischen der deontischen und der epistemischen Modalität ... Selbstverständlich fehlen zweideutige Beispiele gar nicht, in denen die epistemische und die deontische Interpretation gleichzeitig akzeptabel sind. Was das Lateinische charakterisiert, ist die Tatsache, dass manchmal, neben den epistemischen und deontischen Interpretationen, eine evidentiale ebenso annehmbar ist.” Vgl. Bolkestein (1980: p. 5).

54 Plungian (2001: p. 354).

55 Plungian (2010: p. 46).

56 Aikhenvald (2004: p. 19). 


\subsection{Infinitivkonstruktionen, Konjunktiv und Objektsätze nach verba dicendi}

Eines der Hauptmittel zum Ausdruck der indirekten Rede in beiden Werken sind die von verba dicendi abhängigen Infinitivkonstruktionen. Darunter ist Accusativus cum infinitivo (AcI) am meisten vertreten, z.B. (9a-d).

9a) (Trimalchio) ... nihil melius esse dicebat quam sine turba lavari (Petron. Sat. 73,2); "(Trimalchio) ... sagte, nichts sei besser, als ohne Gedränge zu baden."

9b) Negant ... medici sine nervis homines ambulare posse (Petron. Sat. 129,5); "Die Ärzte sagen, dass Leute ohne Sehnen nicht gehen können."

9c) ... (Pythias) iubet officialem suum insuper pisces inscendere ac pedibus suis totos obterere (Apul. Met. 1,25); “... (Pythias) befiehlt seinem Unterbeamten sich auf die Fische hinzustellen und sie mit Füßen zu zertreten.”

9d) Photis ... me accurrit indicatque dominam suam ... nocte proxima in avem sese plumaturam atque ad suum cupitum sic devolaturam (Apul. Met. 3,21); "Fotis huscht zu mir herein und verkündigt mir, ihre Frau würde sich diese Nacht befiedern und zu ihrem Geliebten hinfliegen."

Nominativus cum infinitivo für die Bezeichnung der reportativen Evidentialität kommt nur einmal bei Apuleius (9e) vor.

9e) Nescio ... domina: puto puellam ... Psyches nomine dici: illam dicitur efflicte cupere (Apul. Met. 5,28); "Das weiß ich nicht ... Herrin. Ich glaube aber, dass das Mädchen Psyche heißt; man sagt, dass er es heftig begehrt."

Da solche Konstruktionen von verba dicendi abhängen und den lexikalischen Bestandteil der reportativen Semantik einschließen, ${ }^{57}$ können sie nicht für rein grammatisierte Evidentialien gehalten werden. Doch wenn man in Betracht nimmt, dass sie eine Information aus der zweiten Hand berichten, können sie ohne Zweifel zu den Evidentialitätsstrategien gerechnet werden.

Dem Sinne nach stehen die von verba dicendi abhängigen Objektsätze den Infinitivkonstruktionen nah. Unter ihnen sind in den analysierten Werken Sätze mit Konjunktionen $u t$, quod und indirekte Fragen vorhanden. Dazu kann man auch konjunktionslose Sätze zurechnen, die den Objektsätzen identisch sind, z.B. (10a-d).

10a) (Miles) coepitque hortari lugentem ne perseveraret in dolore supervacuo, ac nihil profuturo gemitu pectus diduceret (Petron. Sat. 111,8); “(Der Soldat) hat angefangen die Trauernde aufzufordern im überflüssigen Schmerz nicht zu verharren und ihr Herz mit unnötigem Wehklagen nicht herunterzubringen."

10b) ... secum eos animadverteram conloquentes quod in proximo nobis esset habenda mansio et totius viae finis quieta eorumque esset sedes illa et habitatio (Apul. Met. 4,5); “... aus ihren Reden habe ich verstanden, dass wir in der Nähe den Aufenthaltsort und ein ruhiges Ende unserer Reise haben sollen, da dort ihr ständiger Wohnort ist."

10c) ... Ceres et Iuno ... quaesiere (Venerem) cur truci supercilio tantam venustatem micantium oculorum coerceret (Apul. Met. 5,31); “... Ceres und Juno ... fragten (Venus), 
warum sie mit zusammengezogenen Brauen die Schönheit ihrer glänzenden Augen einhülle?"

10d) ... praeco pronuntiat, patres in curiam convenirent (Apul. Met. 10,7); “... der Herold ruft aus, die Senatoren sollen in Kuriengebäude zusammenkommen.”

Alle genannten Satztypen sind bei Apuleius vertreten, während Petronius vornehmlich sich mit $u t$-Sätzen bedient.

Der Konjunktiv in solchen Sätzen dient nicht nur als formelles Merkmal der Hypotaxe, sondern auch unterstreicht die Subjektivität und Distanzierung der Aussage und somit stimmt mit der durch Vermittler erhaltenen Information über. ${ }^{58}$

Im folgenden Beispiel (10e), das die Rede des Räubers vor seinen Mitkämpfern darstellt, stehen alle Verben im Infinitiv oder Konjunktiv:

10e) Cognitoque quosdam, immo vero fortissimus quemque variis quidem sed impigris casibus oppetisse, suadet tantisper pacatis itineribus omniumque proeliorum servatis indutiis inquisitioni commilitonum potius insisteretur et tirocinio novae iuventutis ad pristinae manus numerum Martiae cohortis facies integraretur: nam et invitos terrore compelli et volentes praemio provocari posse nec paucos humili servilique vitae renuntiantes et instar tyrannicae potestatis sectam suam conferre malle. Se quoque iam dudum pro sua parte quendam convenisse hominem et statu procerum et aetate iuvenem et corpore vastum et manu strenuum, eique suasisse ac denique persuasisse, ut manus hebetatas diutina pigritia tandem referret ad frugem meliorem bonoque secundae, dum posset, frueretur valetudinis, nec manus validam erogandae stipi porrigeret sed hauriendo potuit exerceret auro (Apul. Met. 7,4).

„Als er hörte, daß einige von ihnen, und zwar die wackersten, bei verschiedenen gefährlichen Vorfällen draufgegangen wären, so machte er den Vorschlag: Man solle eine Weile die Landstraßen in Frieden lassen und allgemeinen Waffenstillstand beobachten; unterdessen aber sich auf Anwerbung junger Leute legen, damit ihr martialisches Heer völlig rekrutiert und wieder so vollzählig als ansehnlich würde. Die Widerspenstigen könne man ja mit Gewalt zwingen und die Unentschlossenen durch Geschenke gewinnen, davon nichts zu erwähnen, dass sehr viele herzlich gern freiwillig zu ihrer Gesellschaft übergehen würden, um sich nur dem Joche der Knechtschaft zu entziehen und bei ihnen ein freies Herrenleben zu führen. Er für sein Teil sei unlängst einem großen, vierschrötigen, handfesten, jungen Kerl begegnet und habe ihm angeraten und endlich ihn auch überredet, die Nerven seines Armes nicht in steter Faulheit erschlaffen zu lassen, sondern zu etwas Besserem anzuwenden, den Vorteil einer festen Gesundheit sich noch zur rechten Zeit zunutze zu machen und nicht schüchtern nach kärglichen Almosen seine starke Faust auszurecken, mit der er gebieterisch große Geldsummen einfordern könnte.“

Mit dem lateinischen Konjunktiv kann man hier den optativus obliquus im Altgriechischen vergleichen, der auch als Evidentialitätsstrategie gemeint werden kann. ${ }^{59}$ Wie das

58 Wolanin (2012: p. 708) charakterisiert einen solchen Konjunktiv als "coniunctivus ex mente aliena."

59 Van Rooy (2016: p. 41): "[optativus obliquus] can also be regarded as an evidential strategy in that the information it expresses is attributed to another center of consciousness, different from the speaker at least in time and possibly also in person.” 
Lateinische benutzte auch das Altgriechische in großem Umfang Infinitivkonstruktionen und Nebensätze als Marker der reportativen Evidentialität.

Die Anwendung der grammatischen Formen mit modaler Bedeutung zum Ausdruck einer fremden Meinung ist auch für moderne Sprachen eigen, z.B. Konjunktiv I im Deutschen oder conditionel im Französischen.

\subsection{Reflexivpronomen sui}

Als Kennzeichen der reportativen Evidentialität in indirekter Rede kann neben den in 3.1. behandelten Mitteln auch das Reflexivpronomen sui in der logophorischen Funkti${ }^{o{ }^{60}}$ auftreten, das die Koinzidenz des Sprechers und des Subjekts der abhängigen Prädikation bezeichnet und sich auf die dritte Person bezieht. Es ist in folgenden Beispielen (11a-d) gezeigt, wo das Reflexivpronomen sich mit Subjekten des Hauptsatzes korreliert und sie als Informationsquelle darstellt.

11a) Eumolpus ... iurat per deos deasque se neque scire quid acciderit, nec ullum dolum malum consilio adhibuisse, sed mente simplicissima et vera fide in navigium comites induxisse... (Petron. Sat. 101,3); "Eumolpus ... schwört bei den Göttern und Göttinnen er habe keine Ahnung davon, was geschehen ist, er habe im Sinn keine schlechte Absicht, er habe ohne Hintergedanke und mit reinem Gewissen die Gefährten auf das Schiff gebracht."

11b) Exspectabam ut pro reliqua insolentia (Trimalchio) diceret sibi vasa Corintho afferri (Petron. Sat. 50,3); "Ich erwartete, dass (Trimalchio) gemäß seiner sonstigen Unverschämtheit sagen würde, ihm würden die Gefäße direkt aus Korinth gebracht."

11c) ... puella ... honesti pastori pollicetur, si sibi praemium decoris addixisset, sese regnum totius Asiae tributuram (Apul. Met. 10,32); “... das Mädchen versprach dem geehrten Hirten, falls er ihr den Preis der Schönheit zuerkennen würde, dass es (ihm) die Herrschaft über ganz Asien schenken wird."

11d) Respondit hortulanus petere se civitatem proximam (Apul. Met. 9,39); "Der Gemüsegärtner erwiderte, dass er in die nächste Stadt hingeht."

Das erwähnte Pronomen tritt bei Petron in dem Objektsatz auf (11e):

11e) ... servus ... procubuit ad pedes ac rogare coepit, ut se poenae eriperemus (Petron. Sat. 30,7); “... fiel uns ein ... Sklave zu Füßen und fing an zu bitten, dass wir ihm seine Strafe entziehen."

Wie R. Kühner betont, "das Reflexiv steht in Beziehung auf das (grammatische oder logische) Subjekt des regierenden Satzes in allen innerlich abhängigen, d. h. solchen

60 C. Hagège, der den Terminus logophor für die Bezeichnung der Quelle der indirekten Rede eingeführt hat, definiert ihn als "une catégorie particulière de substituts, personnels et possessifs, qui réfèrent à l'auteur d'un discours ou à un participant dont sont rapportées les pensées” (Hagège 1974: p. 287). Über den logophorischen Gebrauch der lateinischen Reflexivpronomina siehe Pompei (2002), über die Affinität der Logophorozität und Evidentialität siehe Dimmendaal (2001); Aikhenvald (2004: p. 133); Aikhenvald (2018); Wiemer (2007: p. 230). 
Nebensätzen, welche nicht als Gedanke des Schriftstellers, sondern aus der Seele eines anderen, besonders des Subjekts des Hauptsatzes ausgesprochen werden und daher zugleich auch im Konjunktiv stehen; dasselbe gilt für die Aussagesätze im acc. c. inf. sowie für die ganze oratio obliqua” ${ }^{61}$

\subsection{Kausalsätze mit quod, quia, quoniam}

Unter den Ausdrucksmitteln der reportativen Evidentialität sind auch Kausalsätze mit Konjunktionen quod, quia, quoniam zu nennen. Wie bekannt, kann das Prädikat solcher Sätze sowohl im Indikativ als auch im Konjunktiv stehen. Der Indikativ weist auf einen objektiven Grund hin, der von dem Sprecher ausgeht. Der Konjunktiv dagegen sieht einen indirekten Zugang zur Information, eine Distanzierung davon und somit die Subjektivität voraus. Als Illustration können folgende Sätze (12a-d) dienen.

12a) Iussit illum (fabrum) Caesar decollari: quia enim, si scitum esset, aurum pro luto haberemus (Petron. Sat. 51,6); "Der Kaiser ließ ihm (d.h. dem Handwerker) den Kopf abschlagen, weil wir nämlich Gold für Dreck achten würden, wenn es bekannt geworden wäre."

12b) Indignatus puer, quod (Trimalchio) Scylacem tam effuse laudaret, catellam in terram deposuit hortatusque est ut ad rixam properaret (Petron. Sat. 64,9); "Der Junge, der sich entrüstete, dass (Trimalchio) den Scylax so übermäßig lobte, setzte die Hündin auf die Erde und hetzte sie zur Rauferei (mit dem Hund)."

12c) Amatorem suum, quod in aliam temerasset, unico uerbo mutauit in feram castorem... (Apul. Met. 1,9); "Sie hat ihren Geliebten, der angeblich einer anderen den Hof machte, mit einem einzigen Wort in einen Biber umgewandelt."

12d) (Puella) me ... dirigere dextrorsum magnopere gestiebat, quod ad parentes eius ea scilicet iretur via (Apul. Met. 6,29); "(Das Mädchen) ... versuchte mich mit allen Kräften rechts zu lenken, weil dieser Weg, wie es ihr schien, zu ihren Eltern führte.”

Ein solcher Konjunktiv kann für Hinweis auf den indirekten Zugang zur Information gehalten werden, weil er eine Distanzierung des Sprechers von dem ausgedrückten Gesichtspunkt und die Subjektivität des Letzten unterstreicht. Es scheint, als ob der Sprecher keine Verantwortung für die Richtigkeit des Gesagten übernehme, da er eine fremde Meinung wiedergibt. ${ }^{62}$

\section{Fazit}

Im vorliegenden Aufsatz wurde der Versuch gemacht einige morphologische Formen und syntaktische Konstruktionen in der lateinischen erzählenden Prosa der I.-II. Jhdt. unter dem Gesichtspunkt der Kategorie der Evidentialität zu analysieren. Sie alle

61 Kühner \& Stegmann (1992: p. 606).

62 Plungian (2010: p. 47). 
können als Evidentialitätsstrategien betrachtet werden, da ihre primäre Funktion in der Bezeichnung des Tempus, des Modus, des Genus usw. besteht und Hinweis auf die Informationsquelle bzw. auf den Zugang zur Information als durch Kontext bedingter Nebeneffekt auftritt. In den behandelten Werken von Petron und Apuleius sind acht solche Strategien für den Ausdruck der indirekten Evidentialität festgestellt. Fünf davon beziehen sich auf die inferentive und drei auf die reportative Evidentialität. Verschiedene Häufigkeit der Ausdrucksmittel der genannten Evidentialitätsstrategien bei beiden Schriftstellern (Tabelle 1) wird m. E. durch Kontext und persönliche Präferenzen jedes Autors verursacht.

Tabelle 1. Anwendungshäufigkeit der Evidentialitätsstrategien bei Petron und Apuleius

\begin{tabular}{|c|c|c|c|c|c|c|c|c|}
\hline \multirow{2}{*}{ Autor Evidentialität } & \multicolumn{5}{|c|}{ Inferentive } & \multicolumn{3}{|c|}{ Reportative } \\
\hline & $\mathrm{Ncl}$ & $\begin{array}{l}\text { Coni. } \\
\text { potent. }\end{array}$ & $\begin{array}{l}\text { Perf. } \\
\text { logi- } \\
\text { cum }\end{array}$ & Fut. I & $\begin{array}{l}\text { Debeo+ } \\
+ \text { Inf. }\end{array}$ & $\begin{array}{l}\text { indir. } \\
\text { Rede }\end{array}$ & Sui & $\begin{array}{l}\text { Kausal- } \\
\text { satz mit } \\
\text { Konj. }\end{array}$ \\
\hline Petron & \begin{tabular}{|l|}
8 \\
$42 \%$
\end{tabular} & $\begin{array}{l}8 \\
42 \%\end{array}$ & - & $\begin{array}{l}1 \\
5,5 \%\end{array}$ & \begin{tabular}{|l|}
2 \\
$10,5 \%$
\end{tabular} & $\begin{array}{l}11 \\
46 \%\end{array}$ & $\begin{array}{l}5 \\
21 \%\end{array}$ & $\begin{array}{l}8 \\
33 \%\end{array}$ \\
\hline Apuleius & \begin{tabular}{|l}
4 \\
$20 \%$ \\
\end{tabular} & \begin{tabular}{|l}
10 \\
$50 \%$ \\
\end{tabular} & $\begin{array}{l}2 \\
10 \%\end{array}$ & \begin{tabular}{|l|}
4 \\
$20 \%$ \\
\end{tabular} & - & $\begin{array}{l}35 \\
59 \%\end{array}$ & \begin{tabular}{|l}
8 \\
$14 \%$
\end{tabular} & $\begin{array}{l}16 \\
27 \%\end{array}$ \\
\hline
\end{tabular}

Die weiteren Untersuchungen in dieser Richtung können sich auf die Analyse anderer Ausdrucksmittel der Evidentialität (z.B. lexikalischen) beziehen. Es lohnt sich auch die Evidentialität sowohl in den Werken von verschiedenen Genres, als auch in typologischer Hinsicht auf Grund des Lateinischen und Altgriechischen zu erforschen.

\section{Bibliographie}

\section{Quellenverzeichnis}

Campbell, D. A. (Ed.). (1982). Greek Lyric: Sappho and Alcaeus (Vol. I). Cambridge: Harvard University Press.

Hanson, J. Arthur (Ed.). (1989). Apuleius. Metamorphoses (Vol. I-II). Cambridge, MA. - London: Harvard University Press.

Heinse, W. (Ed.). (1913). Petronius: Das Gastmahl des Trimalchio. Nach dem Satiricon des Petronius übersertzt von Wilhelm Heinse. Düsseldorf: Ernst Ohle.

Kovacs, D. (Ed.). (1995). Euripides: Children of Heracles. Hippolytus. Andromache. Hecuba. Cambridge: Harvard University Press.

Nisard, D. (Ed.). (1865). Pétrone: Apulée, Aulu-Gelle. Euvres complètes. Paris: Didot.

Warmington, E.H. (Ed.). (1987). Titi Petroni Arbitri Satyricon, with an English translation by Michael Heseltine. Seneca: Apocolocyntosis, with an English translation by W. H. D. Rouse. Cambridge, MA: Harvard University Press. 


\section{Sekundärliteratur}

Aikhenvald, A., \& Dixon R. M. W. (1998). Evidentials and areal typology: a case study from Amazonia. Language Sciences, 20, 241-257.

Aikhenvald, A. Y. (2003). Evidentiality in typological perspective. In A. Y. Aikhenvald, \& R. M. W. Dixon (Eds.), Studies in Evidentiality (pp. 1-32). Amsterdam: John Benjamins.

Aikhenvald, A. Y., \& Dixon, R. M. W. (Eds.). (2003). Studies in Evidentiality. Amsterdam: John Benjamins.

Aikhenvald, A. Y. (2004). Evidentiality. Oxford: Oxford University Press.

Aikhenvald, A. Y. (2011). The Grammaticalization of Evidentiality. In H. Narrog, \& B. Heine (Eds.), The Oxford Handbook of Grammaticalization (pp. 605-613). Oxford: Oxford University Press.

Aikhenvald, A. Y. (2018). Evidentiality. The framework. In A. Y. Aikhenvald (Ed.), The Oxford Handbook of Evidentiality (pp. 37-80). Oxford: Oxford University Press.

Anderson, L. B. (1986). Evidentials, Paths of Change, and Mental Maps: Typologically Regular Asymmetries. In W. Chafe, \& J. Nichols (Eds.), Evidentiality: The Linguistic Coding of Epistemology (pp. 273-312). Norwood, NJ: Ablex Publishing Corporation.

Bakker, E. (1997). Storytelling in the Future: Truth, Time, and Tense in Homeric Epic. In E. Bakker, \& A. Kahane (Eds.), Written Voices, Spoken Signs: Tradition, Performance, and the Epic Text (pp. 11-36). Cambridge, MA. - London: Harvard University Press.

Bakker, S. (2002). Futura zonder toekomst. Lampas, 35(3), 199-214.

Bolkestein, A. M. (1980). Problems in the description of modal verbs. An investigation of latin. Assen: Van Gorcum.

Boye, K., \& Harde, P. (2009). Evidentiality: Linguistic Categories and Grammaticalization. Functions of Language, 16(1), 9-43.

Chafe, W. (1986). Evidentiality in English Conversation and Academic Writing. In W. Chafe, \& J. Nichols (Eds.), Evidentiality: the Linguistic Coding of Epistemology (pp. 261-272). Norwood, NJ: Ablex Publishing Corporation.

Chafe, W., \& Nichols, J. (Eds.). (1986). Evidentiality: The Linguistic Coding of Epistemology. Norwood, NJ: Ablex Publishing Corporation.

Comrie, B. (1976). Aspect. Cambridge: Cambridge University Press.

Cornillie, B. (2007a). Evidentiality and Epistemic Modality in Spanish Semi Auxiliaries: A Cognitive-Functional Approach. Berlin - New York: Mouton de Gruyter.

Cornillie, B. (2007b). The continuum between lexical and grammatical evidentiality: a functional analysis of Spanish parecer. Italian Journal of Linguistics, 19(1), 109-128.

Cornillie, B. (2009). Evidentiality and Epistemic Modality: On the Close Relationship between Two Different Categories. Functions of Language, 16(1), 44-62.

Cuzzolin, P. (2010). Evidentialitätsstrategien im Lateinischen. Vorläufige Bemerkungen. In M. Kienpointner, \& P. Anreiter (Eds.), Latin Linguistics Today: Proceedings of the 15. CILL, Innsbruck, April 4-9, 2009 (pp. 247-256). Innsbruck: Universität Innsbruck.

Delbrück, B. (1897). Vergleichende Syntax der indogermanischen Sprachen (Vol. II). Strassburg: Karl J. Trübner. 
Dendale, P. (1993). Le conditionnel de l'information incertaine: marqueur modal ou marqueur evidentiel? In G. Hilty (Ed.), Proceedings of the XXe Congrès International de Linguistique et Philologie Romanes, T. 1, Section 1: La phrase (pp. 165-176). Tübingen: Francke.

Diewald, G., \& Smirnova, E. (Eds.). (2010). Linguistic Realization of Evidentiality in European Languages. Berlin - New York: Mouton de Gruyter.

Diewald, G., \& Smirnova, E. (2010). Introduction. Evidentiality in European Languages: The Lexical-Grammatical Distinction. In G. Diewald, \& E. Smirnova (Eds.), Linguistic Realization of Evidentiality in European Languages (pp. 1-14). Berlin - New York: Mouton de Gruyter.

Dimmendaal, G. J. (2001). Logophoric Marking and Represented Speech in African Languages as Evidential Hedging Strategies. Australian Journal of Linguistics, 21(1), 131-157.

Ernout, A., \& Thomas, F. (1964). Syntaxe latine. Paris: Klincksieck.

Faure, R. (2010). L'optatif oblique serait-il un temps? Lalies, 30, 281-294.

Faure, R. (2014). The Oblique Optative, a Case of Narrative Tense: The Example of the Future Optative. In A. Bartolotta (Ed.), The Greek Verb. Morphology, Syntax, Semantics. Proceedings of the 8th International Meeting of Greek Linguistics (pp. 131-148). Louvain: Peeters.

Fonseca, A. L. (2009). Modo y Modalidad. In M. Baños Baños (Ed.), Sintaxis del latín clásico (pp. 443-468). Madrid: Liceus.

Friedman, V. A. (1986). Evidentiality in the Balkans: Bulgarian, Macedonian, and Albanian. In W. Chafe, \& J. Nichols (Eds.), Evidentiality: The Linguistic Coding of Epistemology (pp. 168-187). Norwood, NJ: Ablex Publishing Corporation.

Givon, T. (1982). Evidentiality and Epistemic Space. Studies in Language, 6(1), 23-49.

Greco, P. (2013). Latin Accusativus cum Participio: Syntactic Description, Evidential Values, and Diachronic Development. Journal of Latin Linguistics, 12(2), 173-198.

Guardamagna, C. (2017). Reported Evidentiality, Attribution and Epistemic Modality: A Corpus-based Diachronic Study of Latin secundum NP (according to NP). Language Science, 59, 159-179.

Guentchéva, Z. (1994). Manifestation de la cathégorie médiatif dans les temps du français. Langue français, 102, 8-23.

de Haan, F. (1999). Evidentiality and Epistemic Modality: Setting Boundaries. Southwest Journal of Linguistics, 18, 83-101.

de Haan, F. (2007). Raising as grammaticalization: the case of Germanic seem-verbs. Italian Journal of Linguistics, 19(1), 129-150.

Hagège, C. (1974). Les pronoms logophoriques. Bulletin de la Société de Linguistique de Paris, 69, 287-310.

Hansen, B. (2007). Evidencialnost v nemeckom yazyke [Evidentiality in German]. In V. S. Khrakovskiy (Ed.), Evidencialnost v yazykakh Europy $i$ Azii [Evidentiality in the Languages of Europe and Asia] (pp. 241-252). Sankt-Petersburg: Nauka.

Hennemann, A. (2013). A Context-Sensitive and Functional Approach to Evidentiality in Spanish or Why Evidentiality Needs a Superordinate Category. Frankfurt am Main: Peter Lang.

Jakobson, R. (1984). Shifters, Verbal Categories, and the Russian Verb. In L. R. Waugh, \& M. Hall (Eds.), Russian and Slavic Grammar Studies 1931-1981 (pp. 41-58). Berlin - New York - Amsterdam: Mouton Publishers. 
Joseph, B. D. (2003). Evidentiality in Proto-Indo-European? Building a Case. In K. Jones-Bley, M. Huld, A. Della Volpe, \& M. Robbins Dexter (Eds.), Proceedings of the Fourteenth Annual UCLA Indo-European Conference (pp. 96-111). Washington, DC: Institute for the Study of Man.

Khrakovskiy, V. S. (Ed.). (2007). Evidencialnost v yazykakh Europy $i$ Azii [Evidentiality in the Languages of Europe and Asia]. Sankt-Petersburg: Nauka.

Kozinceva, N. A. (2007). Tipologiya kategorii zasvidetelstvovannosti [Typology of Category of Evidentiality]. In V. S. Khrakovskiy (Ed.), Evidencialnost v yazykakh Europy i Azii [Evidentiality in the Languages of Europe and Asia] (pp. 13-36). Sankt-Petersburg: Nauka.

Kratschmer, A., \& Heijnen, A. (2010). Revelative Evidentiality in European Languages: Linguistic Marking and Its Anthropological Background. In G. Diewald, \& E. Smirnova (Eds.), Linguistic Realization of Evidentiality in European Languages (pp. 331-368). Berlin - New York: Mouton de Gruyter.

Kühner, R., \& Stegmann, C. (1992). Ausführliche Grammatik der lateinischen Sprache, 3.1: Satzlehre. Hannover: Hahn.

Lampert, G., \& Lampert, M. (2010). Where Does Evidentiality Reside? Notes on (Alleged) Limiting Cases: seem and be like. STUF - Language Typology and Universals, 63(4), 308-321.

Lazard, G. (2001). On the Grammaticalization of Evidentiality. Journal of Pragmatics, 33, 359-367.

Méndez Dosuna, J. V. (1999). La valeur de l'optatif oblique grec: Un regard fonctionnel-typologique. In B. Jacquinod (Ed.), Les complétives en grec ancien: Actes du colloque international de Saint-Étienne (pp. 331-352). Saint-Étienne: Publications de l’Université de Saint-Étienne.

Mišeska Tomić, O. (2008). An Integrated Areal-Typological Approach: Local Convergence of Morphosyntactic Features in the Balkan Sprachbund. In P. Muysken (Ed.), From Linguistic Areas to Areal Linguistics (pp. 181-219). Amsterdam - Philadelphia: John Benjamins.

Mushin, I. (2001). Evidentiality and Epistemological Stance: Narrative Retelling. Amsterdam - Philadelphia: John Benjamins.

Pinkster, H. (1986). Three notes on the Latin subjunctive. Revue Informatique et Statistique dans les Sciences Humaines, 22, 147-156.

Pinkster, H. (2015). The Oxford Latin Syntax, Vol. I: The Simple Clause. Oxford: Oxford University Press.

Plungian, V. A. (2001). The Place of Evidentiality within the Universal Grammatical Space. Journal of Pragmatics, 33, 349-357.

Plungian, V. A. (2010). Types of Verbal Evidentiality Marking: An Overview. In G. Diewald, \& E. Smirnova (Eds.), Linguistic Realization of Evidentiality in European Languages (pp. 15-58). Berlin - New York: Mouton de Gruyter.

Pompei, A. (2002). Riflessivi indiretti in latino e logoforicita. Studi Italiani di Linguistica Teorica e Applicata, 2002, 398-446.

van Rooy, R. (2016). The Relevance of Evidentiality for Ancient Greek: Some Explorative Steps through Plato. Journal of Greek Linguistics, 16(1), 3-46.

Serbat, G. (1980). Le parfait de l'indicatif actif en latin. In G. Serbat (Ed.), Le sens du parfait de l'indicatif actif en Latin (pp. 12-54). Paris: Université de Paris-Sorbonne.

Squartini, M. (2001). The internal structure of evidentiality in Romance. Study in Language, 25, 255-296. 
Squartini, M. (2004). Disentangling evidentiality, and epistemic modality in Romance. Lingua, $114(7), 873-895$.

Squartini, M. (2008). Lexical vs. grammatical evidentiality in French and Italian. Linguistics, 46(5), 917-947.

Wiemer, B. (2007). Kosvennaya zasvidetelstvovannost v litovskom yazyke [Indirect evidentiality in Lituanian]. In V. S. Khrakovskiy (Ed.), Evidencialnost v yazykakh Europy i Azii [Evidentiality in the Languages of Europe and Asia] (pp. 197-240). Sankt-Petersburg: Nauka.

Wiemer, B. (2010). Hearsay in European Languages: Toward an Integrative Account of Grammatical and Lexical Marking. In G. Diewald, \& E. Smirnova (Eds.), Linguistic Realization of Evidentiality in European Languages (pp. 59-129). Berlin - New York: Mouton de Gruyter.

Wiemer, B. (2017). Reliability as an Intermediate Layer between Evidential and Epistemic Meanings. In Book of Abstracts. 50th Annual Meeting of the Societas Linguistica Europaea, 10-13 September 2017, University of Zurich, Switzerland (pp. 666-667) [retrieved 05.05.2019 from http://www. sle2017.eu/downloads/BOOK\%20OF\%20ABSTRACTS\%205-7-2017.pdf].

Willett, Th. L. (1988). A Cross-Linguistic Survey of the Grammaticization of Evidentiality. Studies in Language, 12(1), 51-97.

Wolanin, H. (2012). Grammatyka opisowa klasycznej taciny w ujęciu strukturalnym. Kraków: Księgarnia Akademicka.

Zheltova, E. V. (2017). Evidential Strategies in Latin. Hyperboreus, 23(2), 313-337.

Zheltova, E. V. (2018). How to Express Surprise without Saying "I'm Surprised" in Latin. Philologia Classica, 13(2), 228-240.

Bohdan Chernyukh, PhD / cherboh@gmail.com

Professor at Department of Classical Philology

Ivan Franko National University of Lviv

Universytetska 1, 79000 Lviv, Ukraine

Toto dílo Ize užít v souladu s licenčními podmínkami Creative Commons BY-SA 4.0 International (https://creativecommons.org/licenses/by-sa/4.0/legalcode). Uvedené se nevztahuje na díla či prvky (např. obrazovou či fotografickou dokumentaci), které jsou v díle užity na základě smluvní licence nebo výjimky či omezení př́slušných práv. 